\title{
Le pari de la durabilité à Sotchi sur le littoral russe de la mer Noire
}

Ekaterina Andreeva-Jourdain

\section{(2) OpenEdition}

12 Journals

Édition électronique

URL : http://journals.openedition.org/tourisme/1176

DOI : 10.4000/tourisme.1176

ISSN : 2492-7503

Éditeur

Éditions touristiques européennes

Référence électronique

Ekaterina Andreeva-Jourdain, « Le pari de la durabilité à Sotchi sur le littoral russe de la mer Noire », Mondes du Tourisme [En ligne], Hors-série | 2016, mis en ligne le 01 septembre 2016, consulté le 02 mai 2019. URL : http://journals.openedition.org/tourisme/1176; DOI : 10.4000/tourisme.1176

Ce document a été généré automatiquement le 2 mai 2019.

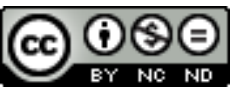

Mondes du tourisme est mis à disposition selon les termes de la licence Creative Commons Attribution - Pas d'Utilisation Commerciale - Pas de Modification 4.0 International. 


\title{
Le pari de la durabilité à Sotchi sur le littoral russe de la mer Noire
}

\author{
Ekaterina Andreeva-Jourdain
}

1 Les côtes de la mer Noire étaient depuis le XIX ${ }^{\mathrm{e}}$ siècle un lieu de villégiature privilégié des élites russes et des soviétiques. Mais les difficultés économiques de cette région après la chute de l'Union des républiques socialistes soviétiques (URSS) et la forte attractivité d'autres destinations plus exotiques ont détourné, pour un certain temps, les touristes russes de la mer Noire. La pratique de départs en vacances dans des pays chauds (Turquie, Égypte, Thailande, Émirats arabes, etc.) est devenue assez courante depuis 1992 dans tous les segments de la population russe, principalement auprès des jeunes et des familles avec enfants (Andreeva, 2006).

2 Cependant depuis quelques années, la fréquentation touristique du littoral russe se renforce de manière considérable et connaît une augmentation qui varie entre 10 et $15 \%$ par an. Selon les statistiques officielles de l'État, $20 \%$ de tous les flux touristiques intérieurs se dirigent vers les côtes de la mer Noire. La ville de Sotchi est la principale station balnéaire de cette zone (Agence fédérale du tourisme de la Fédération de Russie, 2012).

3 La recherche universitaire russe actuelle s'est principalement focalisée sur l'étude du tourisme international dans le pays, effectuée sous le prisme de son impact sur l'économie de la Russie (la part de l'industrie du tourisme dans le Produit intérieur brut (PIB) du pays). Les chercheurs évoquent souvent le problème du développement du tourisme extérieur russe après la chute de l'URSS et les conséquences que cela représente pour l'économie russe. Ils négligent souvent les aspects sociaux et la dimension spatiale du phénomène touristique. Dans les années 2000, des chercheurs comme Claude Cabanne et Elena Tchistiakova (2002) ont essayé de dresser un état des lieux des transformations socio-économiques connues après la chute de l'URSS dans le champ du tourisme côtier en Russie, y compris sur le littoral de la mer Noire. Actuellement, c'est principalement la question de l'impact des Jeux olympiques d'hiver de 2014 sur le développement du 
tourisme dans la région qui suscite un intérêt particulier de la part des chercheurs russes et étrangers ${ }^{1}$.

4 Cet article a pour objectif d'apporter des réponses à la question suivante : comment la station balnéaire de Sotchi est-elle née, comment évolue-t-elle, répond-elle à la durabilité du tourisme?

5 Le choix de la ville de Sotchi pour cette étude est justifié par le fait qu'elle reflète au niveau régional les effets de la politique touristique nationale, et le rôle que prend l'État en termes de législation, de politique d'accueil et de prise en charge des infrastructures.

6 Après un rapide état des lieux du tourisme intérieur russe, cet article se penchera sur un cas particulier de la station balnéaire de Sotchi, située au bord de la mer Noire, dans la partie russe du Caucase. L'analyse de la construction de la pérennité du lieu par une approche géo-historique sera suivie de l'étude des transformations de la station effectuée sous le prisme des nouvelles formes de tourisme et des investissements des acteurs publics et privés.

7 Cet article est basé sur les résultats d'un travail de terrain effectué à Sotchi pendant l'été 2012. Pour l'analyse historique de la mise en tourisme de la station, nous avons eu recours aux archives d'État et à celles des syndicats. L'étude des aménagements et du développement touristique de la station après la chute de l'Union soviétique a été effectuée à l'aide du dépouillement des rapports de fréquentation touristique produite chaque année par le Comité du tourisme de Sotchi. La méthode d'observation a été employée pour l'analyse des pratiques spatiales des Russes à Sotchi. Enfin, nous nous sommes appuyés sur l'analyse de la presse locale, qui reflète bien la situation actuelle en matière d'aménagement touristique du territoire étudié.

\section{Le renouveau du tourisme intérieur russe}

8 Le tourisme extérieur a été le premier à être développé dans la société russe après la chute de l'Union soviétique en 1991. Cependant depuis quelques années, les touristes nationaux sont de plus en plus nombreux à vouloir rester en Russie pour les vacances, provoquant par ce fait le regain du tourisme intérieur en Russie. Le littoral joue un rôle important dans la structuration des pratiques touristiques des Russes, du fait que le concept de vacances au sein de cette nation reste avant tout associé à la notion de repos, surtout dans leur propre pays.

\section{Le tourisme intérieur russe, une affaire d'État}

Le tourisme des Russes en Russie est un phénomène méconnu et pourtant en pleine croissance. Selon les sources officielles, le nombre de touristes intérieurs connait une augmentation annuelle constante qui varie entre 4 et $8 \%$ depuis 2007 et a atteint en 2011 la barre de 36 millions de personnes (Agence fédérale du tourisme de la Fédération de Russie, 2012). Le tourisme intérieur domine de loin le tourisme international en Russie, qui compte 2,3 millions d'individus pour l'année 2011 (Rosstat, Service fédéral des statistiques). 
Tableau 1. La dynamique des flux touristiques intérieurs en Russie (2007-2011) en millions de personnes

\begin{tabular}{|l|l|l|l|l|l|}
\hline Année & 2007 & 2008 & 2009 & 2010 & 2011 \\
\hline Nombre de touristes en millions de personnes & 26,6 & 28,7 & 31,7 & 34,7 & 36,0 \\
\hline
\end{tabular}

Source : Agence fédérale du tourisme de la Fédération de Russie, 2012. Élaboration propre.

10 L'une des raisons du développement du tourisme intérieur est l'accroissement de la "classe moyenne russe ${ }^{2}$ », principalement dans les grandes villes russes - y compris quinze villes de plus d'1 million d'habitants -, ce qui entraîne une augmentation du nombre de touristes portant de plus en plus d'intérêt à leur propre pays. En effet, depuis la chute de l'Union soviétique les grandes villes russes se trouvent au centre des transformations économiques et sociales, avec l'affirmation d'une société de consommation associée à une sphère des loisirs.

11 La politique actuelle du président de la Fédération de Russie Vladimir Poutine vise à augmenter le tourisme intérieur en Russie. C'est à partir des années 2000 que le gouvernement commence à intégrer dans ses plans de développement touristique, conçus pour une période de 7 ans, l'existence d'un marché local de proximité. Le « Programme de développement du tourisme de la Fédération de Russie pour la période 2011-2018 ", lancé en mars 2011, a pour objectif une augmentation considérable du nombre de touristes intérieurs, qui devrait atteindre, selon les pronostics de l'État russe, 45 millions de personnes en 2018.

12 L'année 2005 marque un tournant important car le gouvernement russe a mis en place des jours fériés payés en début d'année (durant la période du $1^{\mathrm{er}}$ au 10 janvier), destinés aux travailleurs afin de développer une économie de loisirs au sein du pays. Ce principe d'attribution de jours fériés rémunérés renforce le système bien établi du week-end et des vacances dans les entreprises publiques et privées, avec l'augmentation du nombre de jours de congés annuels en fonction de l'ancienneté. C'est le premier engagement fort de l'État incitant à l'émergence d'une économie des loisirs. Selon les statistiques officielles, cette mesure provoque une augmentation considérable (entre 15 et $20 \%$ par an) de touristes intérieurs restant au sein de leur pays pour les vacances de fin d'année.

En 2012, l'État russe décide d'écourter la période de jours fériés en janvier (le nombre de jours fériés passe de 10 à 8 à partir du mois de janvier 2013) au profit de congés plus longs au mois de mai (du $1^{\text {er }}$ au 3 mai). Le gouvernement russe espère encourager des départs touristiques moins éloignés mais plus fréquents.

14 Enfin, en 2005 le gouvernement russe a choisi le mécanisme des zones économiques spéciales (ZES) (Loi fédérale du 22 juillet 2005, N116-F3, « Les zones économiques spéciales en Fédération de Russie »). La gestion de ces zones a été confiée à la société étatique de gestion des ZES en 2006. Il s'agit de dix-sept zones du réseau fédéral RosZES sur lesquelles sont accordés des privilèges exclusifs aux entreprises spécialisées dans différents secteurs d'activité. L'État ne prélèvera ni impôts sur les sociétés ni taxes sur les transports pendant les dix premières années, ni impôts fonciers pendant cinq ans, aucune TVA ne sera appliquée sur les équipements importés sur ces territoires. Le gouvernement prévoyait des investissements de plus de 330 milliards de roubles (8 milliards d'euros). Quatre types de zones de ce genre fonctionnent : industrielles, d'application de nouvelles 
technologies, de récréation et de tourisme, ainsi que portuaires et logistiques. Initialement, l'État a ciblé huit zones touristiques spéciales en tant que hauts lieux du tourisme à l'échelle du pays. Elles sont situées dans la région de Stavropol (Yutsa), de Kaliningrad, d'Irkoutsk, de l'Altaï (Katun et Altaï Valley), de la république de Bouriatie (Baïkal Haven), de Primoriye (Russky Island) et du Caucase du Nord (carte 1). Il s'agit des territoires qui se situaient dans les régions russes populaires à l'époque soviétique, comme c'est le cas de la région du Caucase du Nord, du lac Baïkal ou des montages de l'Altaï. Créées pour une période de 20 ans maximum, ces zones couvrent les territoires spécialisés dans différents types de tourisme : dans l'Altaï, le gouvernement envisage de développer le thermalisme, le tourisme sportif et d'aventure (rafting, ski alpin, randonnées équestres). Dans la région d'Irkoutsk, l'État mise sur la progression des voyages d'affaires, en Bouriatie sur le ski alpin et les sports aquatiques. La région du Caucase du Nord abritant les plus anciennes villes balnéaires de la Russie bénéficie d'une approche spécifique de la part du gouvernement russe. Un cluster touristique est en création sur son territoire sous la gestion d'une autre société d'état «Les stations du Caucase du Nord».

Carte 1. Les zones économiques spéciales de tourisme en Russie en 2010

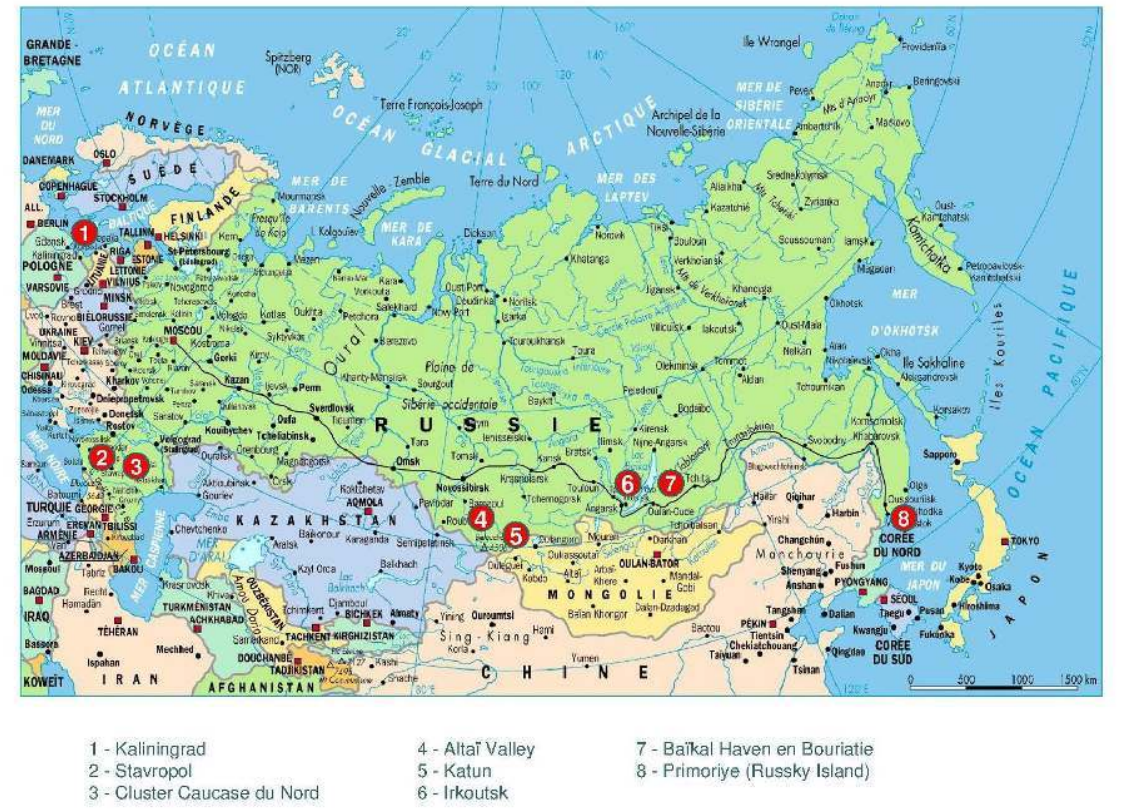

Conception et réalisation : E. Andreeva-Jourdain (2014).

Il existe une vraie filiation entre le tourisme domestique en Union soviétique et la période contemporaine dans le choix des espaces touristiques du pays. L'État russe mise sur des valeurs sûres en essayant de relancer les formes traditionnelles du tourisme répandues dans la société depuis l'époque soviétique. Le gouvernement de Vladimir Poutine tente de sensibiliser la population par rapport au tourisme domestique en l'ancrant dans une tradition nationale. Ce fait souligne le rôle de l'État dans le contrôle du contenu du tourisme au niveau national.

Néanmoins, les ZES touristiques rencontrent un certain nombre de problèmes depuis leur création en 2005. Pour le début de l'année 2014, ces ZES ont attiré seulement 38 
investisseurs et n'ont assuré la création que de 151 emplois (Zoubareva, 2014). Selon les dires du directeur général de la Société de gestion des zones économiques spéciales, M. Vadim Tretiakov, elles se situent parmi les moins efficaces des zones, ce qui est dû au manque d'expérience dans le processus de développement territorial et à leur spécificité qui comprend entre autres la nécessité d'importants investissements dans l'infrastructure (Tretiakov, 2014). L'État russe a préféré miser sur des territoires connus depuis l'époque soviétique pour leur ressources naturelles sans faire appel à des experts des cabinets de conseil spécialisés dans le diagnostic territorial en tourisme. De plus, un investisseur potentiel n'a pas de pouvoir décisif concernant la réalisation d'un projet dans le cadre de ce dispositif. Le "partenariat public-privé " n'est pas encore très bien développé en Russie. L'on peut évoquer un certain manque de confiance du secteur privé vis-à-vis de l'État. Comme résultat, à ce jour ces territoires n'ont pas attiré un grand nombre d'investissements. Par conséquent, deux zones - celles de Kaliningrad et de Krasnodar («La nouvelle Anapa») dans la région du Caucase du Nord - ont déjà été fermées en raison de l'absence d'investisseurs. La ZES de Primoriye (Russky Island), créée en mars 2010, est sur le point d'être fermée également, car selon la législation russe, une ZES n'ayant pas attiré d'investisseurs 36 mois après sa création peut être fermée (Loi fédérale du 22 juillet 2005, N116-F3, «Les zones économiques spéciales en Fédération de Russie »). Enfin, depuis le 27 décembre 2012, la zone Yutsa située dans la région de Stavropol a été incluse dans le cluster touristique du Caucase du Nord (Arrêté $n^{\circ} 833 \mathrm{du}$ gouvernement de Fédération de Russie du 14 octobre 2010).

Il est important de rappeler qu'il avait déjà existé une tentative de création des ZES en Russie dans les années 1991-1998. L'importance de ces dernières était différente, allant de sites locaux aux municipalités et aux sujets entiers de la Fédération de Russie. La pratique a démontré leur inefficacité, et la plupart des zones sont devenues des offshores.

Ces arguments nous permettent d'émettre l'hypothèse que ces ZES touristiques auront plus de chance de développement en tant que stations d'envergure régionale, et non internationale, ce qui contribuera davantage à l'augmentation des mobilités de faible amplitude.

\section{Les flux du tourisme intérieur russe : où vont les Russes?}

Quant les Russes font du tourisme dans leur pays, ils se déplacent premièrement dans les grandes villes dans la partie européenne de la Russie (Saint-Pétersbourg, Moscou et les villes de l'Anneau d'or ${ }^{3}$ ). Les zones urbaines sont les principaux sites visités, les touropérateurs proposent des visites culturelles, ce qui correspond à la pratique de découverte telle qu'elle a été décrite par l'équipe Mit (2002). Les Russes veulent découvrir ou redécouvrir le patrimoine historique et culturel que la période soviétique a pu effacer de leur mémoire. En effet, les dirigeants de l'Union soviétique désapprouvaient fortement les monuments se rapportant à l'ancien régime tsariste et à la religion, et ne souhaitaient pas que ses concitoyens les visitent ou leur portent trop d'intérêt. Lors de leurs voyages de découverte, les touristes russes visitent des temples, des églises, des cathédrales, ils effectuent également le tour de villes dont le but principal est d'observer l'architecture locale, ce qui leur permet de réapprendre l'histoire du pays à travers ces visites.

Les nombreuses rivières qui traversent les régions du pays ont permis l'essor du tourisme fluvial, dont l'offre est abondante. La Volga, l'Ienisseï, la Léna font partie de l'offre de croisières. Les activités sportives comme le rafting et le kayak sont encore en 
développement et leur offre est aujourd'hui représentée au Caucase, dans l'Altaï, dans la république de Carélie et au Baïkal. Les activités moins extrêmes comme le catamaran et la planche à voile sont proposées actuellement dans les républiques d'Adyguée, de Khakassie et de Saha, mais aussi dans la région d'Amour. Le yachting peut se pratiquer sur les mers Baltique, d'Azov ou de Barents.

Le tourisme d'aventure est en plein développement en Russie. Les activités touristiques de montagne - randonnée, alpinisme - sont renommées. L'offre est en ce domaine immense sur tout le territoire de la Russie : les montagnes du Caucase, l'Altaï, l'Oural du Sud, la Sibérie et le Kamtchatka.

Le trekking est une pratique populaire en Russie. Les randonnées organisées sont développées majoritairement dans les régions montagneuses: Caucase, régions de Mourmansk, Irkoutsk, Astrakhan, etc.

Pour les adeptes de l'écotourisme, l'offre est très variée en Russie en fonction de ses régions. En premier lieu, il est important de mentionner la république de Carélie, la région des forêts et des lacs, autrement appelée «les poumons de la Russie». Le lac mondialement connu par ses eaux pures et ses dimensions est le Baïkal, se trouvant dans la partie Est de la Sibérie.

Enfin, on peut constater l'apparition du tourisme rural, dont l'offre est la plus développée aujourd'hui dans la région de Pskov. C'est à cet endroit que l'on retrouve encore des villages traditionnels dont les habitants sont croyants et pratiquent des activités d'antan.

Les destinations estivales au sein de la Russie sont quant à elles plus orientées vers le Sud du pays. Ce sont les côtes de la mer Noire, et principalement les régions de Krasnodar et de Sotchi, qui sont les plus prisées par les touristes. Ces séjours touristiques appellent principalement au tourisme balnéaire et curatif, et par extension à la pratique de repos telle que définie par l'équipe Mit (équipe Mit, 2002). Le surf devient de plus en plus populaire aujourd'hui en Russie, en particulier dans la région de Krasnodar. En ce qui concerne la plongée sous-marine, cette activité est toujours pratiquée dans les stations littorales de la mer Noire (Sotchi, Anapa, Touapsé).

La politique de l'État visant le développement du tourisme intérieur en Russie semble pour l'instant atteindre son but, puisque, selon les statistiques officielles, environ 7,1 millions de touristes intérieurs se rendent dans la région de Krasnodar sur les rives de la mer Noire chaque année. Il s'agit principalement d'urbains de la classe moyenne partant en vacances vers les plages de leur pays pour se reposer et profiter des bienfaits de la mer Noire.

\section{Sotchi, une station balnéaire incontournable dans le tourisme national russe}

La ville de Sotchi, qui compte en 2013368011 habitants (445 209 habitants avec l'agglomération), est située au bord de la mer Noire, elle est bordée au nord-est, à l'est et au sud-est par les crêtes du Caucase. Sotchi se trouve à $170 \mathrm{~km}$ au sud-est de Krasnodar et à $1360 \mathrm{~km}$ au sud de Moscou. L'agglomération s'étend sur $145 \mathrm{~km}$ de côtes. Située sur la même latitude que la Côte d'Azur, et bénéficiant de l'abri que constitue le Caucase contre les entrées d'air en provenance du Nord et de Sibérie, Sotchi jouit d'un climat très doux. La ville bénéficie d'un climat subtropical humide, comme toute la zone côtière bordant la mer Noire. 


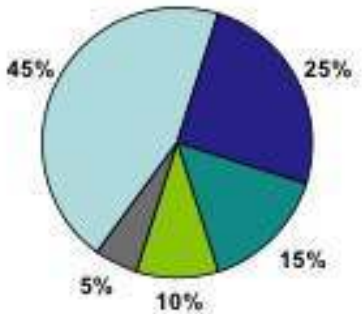

\section{La clientèle touristique de la station} constituent $95 \%$ de touristes.

La ville de Sotchi est le principal pôle touristique du littoral de la mer Noire. Selon les statistiques de l'État, environ 4 millions de touristes russes s'y rendent chaque année, majoritairement l'été. L'objectif principal de la stratégie du développement de la ville de Sotchi est d'attendre 8 millions de touristes par an en 2020.

Malgré le fait que les tarifs des prestations touristiques proposées par cette station dépassent en moyenne 1,5 fois ceux pratiqués par d'autres villes balnéaires russes, la ville de Sotchi reste la station la plus courue de Russie. L'on peut dire qu'il s'agit ici d'une certaine distinction sociale chez les citoyens russes.

La ville de Sotchi subit une concurrence assez modérée de la part des autres stations balnéaires du littoral de la mer Noire comme Touapsé, Guélendjik ou Anapa car la saison dans les deux premières commence et finit assez tôt (début de saison en juin et la fin au moins d'août). La ville d'Anapa est principalement spécialisée dans le repos des enfants (elle possède un grand nombre de colonies de vacances), la demande y est donc très dépendante du calendrier scolaire (Bokov et alii, 2012, p. 76).

1 Sotchi reste une station essentiellement fréquentée par les Russes. En 2012, ces derniers

\section{Fig. 1. Provenance des touristes à Sotchi en 2012}

\section{Source : Comité du tourisme de Sotchi. Élaboration propre.}

Une majeure partie de ces touristes (70\%) viennent de Moscou, Saint-Pétersbourg, Rostov, Stavropol ainsi que de la région de Krasnodar. $25 \%$ des Russes sont originaires des villes de la Sibérie et de la Volga ainsi que du reste du pays. L'on décèle ici le facteur de la proximité du passage de la ville ou de la montagne à la mer. Une comparaison entre les Moscovites qui se rendent à Sotchi et la clientèle parisienne qui part en vacances dans le sud de la France pour le tourisme balnéaire est possible. Les touristes étrangers ne représentent qu'entre 3 et $5 \%$ de la totalité des touristes balnéaires de Sotchi. Il s'agit ici principalement des Biélorusses et des Ukrainiens. Les touristes venant des pays étrangers hors CEI représentent seulement 0,9\% (Bokov et alii, 2012, p. 72). La part des touristes étrangers n'a jamais été élevée à Sotchi. Même pendant la fréquentation maximale en 1985 (260 000 visiteurs non soviétiques), les étrangers n'ont jamais dépassé $10 \%$ de l'ensemble des touristes (Cabanne, 2002, p. 240). Cela s'explique par le fait que les touristes occidentaux visitent principalement Moscou, Saint-Pétersbourg ainsi que les villes de l'Anneau d'or, venant pour découvrir la Russie plutôt que pour se reposer. Par 
ailleurs, la Russie est un pays nordique. Il est donc rare que les Occidentaux se la représentent en tant que «paradis tropical». Enfin, la qualité médiocre des infrastructures ne permet pas pour l'instant de proposer des prestations de haute qualité. Le tourisme individuel a repris à Sotchi en 1999. Depuis les années 2000, la part de voyages individuels est en augmentation (Bokov et alii, 2012, p. 75). Selon les statistiques de l'État, parmi les 7,1 millions de touristes intérieurs se rendant annuellement dans la région de Krasnodar, 4,5 millions organisent le voyage de façon individuelle et 2,6 millions achètent un voyage organisé payé de manière individuelle. Les touristes individuels sont majoritairement des jeunes actifs de 25 à 40 ans qui viennent à Sotchi avec leurs enfants. La ville de Sotchi est donc une station familiale : plus de la moitié des vacanciers arrivent en famille. À la différence des sociétés d'Europe occidentale où les plus de 60 ans constituent une clientèle importante des agences de voyages, les retraités russes sont peu nombreux à voyager en général, ce qui correspond aux difficultés financières de cette catégorie sociale en Russie. On compte entre 2 et $3 \%$ des touristes âgés à Sotchi.

Fig. 2. Diversification des touristes russes venant à Sotchi en 2012 par type de voyage
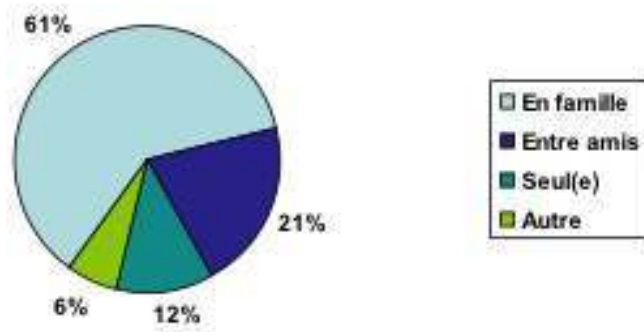

Source : Comité du tourisme de Sotchi. Élaboration propre.

Selon les données du secteur hôtelier, les touristes viennent à Sotchi, en moyenne, pour une durée de 7 à 14 jours. Ceux qui séjournent dans les sanatoriums y restent pour une période de 21 jours, ce qui correspond à la durée d'une cure classique. La majorité des touristes $(73 \%)$ viennent en train. Cela s'explique par l'origine des touristes et par la popularité du train en Russie. $12 \%$ de Russes utilisent l'avion. La voiture individuelle compte encore peu vu l'éloignement important de certains touristes venant, par exemple, des villes de Sibérie.

\section{La construction de la pérennité du lieu}

Le tourisme en Russie, par son histoire et sa politique a d'abord été national, intérieur. Le littoral de la mer Noire, doté d'une tradition touristique forte pendant les époques tsariste et soviétique, représente une sorte de laboratoire des transformations permettant d'étudier la pérennité dans le temps d'un lieu touristique comme Sotchi. 


\section{Sotchi, une station de cure pour les élites avant tout}

38 Les côtes de la mer Noire étaient depuis le XIX siècle un lieu de villégiature privilégié des élites russes. L'histoire de la station balnéaire de Sotchi commence par le travail de la commission spécifique chargée d'explorer le littoral de la mer Noire (de Novorossisk à Soukhoumi) en 1898. Le rapport de cette commission a confirmé les qualités thérapeutiques et climatiques exceptionnelles de la ville. À partir de 1902, l'aristocratie de Saint-Pétersbourg et les industriels de Moscou commencent à s'intéresser à Sotchi. Cela commence par la construction des premières villas et hôtels particuliers.

À cette époque, la ville avait un avenir prometteur et était reconnue par des scientifiques comme Alexandre Voéikov, célèbre climatologue et géographe russe, ou Fédor Pasternatski, clinicien et climatologue. Sotchi était déjà comparée aux stations de cure françaises et italiennes, notamment par le géographe et botaniste russe Andrei Krasnov.

En 1901, la Société du tourisme est créée, son rôle est d'organiser des voyages en Russie et à l'étranger. C'est à partir de cette période que commencent le développement des infrastructures touristiques dans les stations thermales, les premiers hôtels et restaurants.

41 Le premier complexe hôtelier, «La Riviera du Caucase », qui comprennait un hôtel, un théâtre, un casino ainsi qu'un petit établissement de bains, a vu le jour en 1909. En 1913, l'établissement, victime de son succès, comptait déjà 4 hôtels avec une capacité totale de 250 chambres. La ville de Sotchi commence à être à la mode pour les cures thermales. La station fait concurrence aux villas de la Côte d'Azur. 18000 personnes viennent se reposer à Sotchi tous les ans au début du xxe siècle (Bokov et alii, 2012, p. 54).

Quant à l'élite politique russe, c'est la Crimée qui était son endroit de repos préféré au cours du xxe siècle. La presqu'île accueillait la résidence d'été du tsar Nicolas II, la datcha officielle de Josef Staline, les résidences des secrétaires généraux du parti communiste, comme Léonid Brejnev et Nikita Khrouchtchev.

\section{Quand tourisme rime avec mérite}

43 Les préoccupations sociales du régime communiste ont donné naissance à des lieux touristiques multiples, plus particulièrement en Crimée ou dans le Caucase, où les ministères soviétiques, les municipalités, les usines, les sovkhozes ${ }^{4}$ ou les kolkhozes ${ }^{5}$ possédaient des établissements. Ce nouveau modèle touristique s'appuie sur la planification et est basé sur les décrets de Lénine de 1919 concernant « les régions de cure d'importance nationale » (Cabanne, 2002, p. 236).

À partir de 1921 les villas et les hôtels sont nationalisés et transformés par la suite en établissements de cure et de repos dévolus aux kolkhozes et sovkhozes. En 1933 est adopté le plan directeur de la station de cure Sotchi-Matsesta située sur les bords de la mer Noire. Progressivement six stations touristiques seront créées sur $145 \mathrm{~km}$ le long de la mer Noire, de Lazarevskoe au nord-ouest à Adler au sud-est (Cabanne, 2002, p. 236).

En 1913, on comptait 10000 visiteurs des pensions assurant bains et soins thermaux de Sotchi. Dans les années 1930, ils sont 140000 (équipe Mit, 2011, p. 140). Les syndicats soviétiques sont à l'origine du développement touristique rapide des années 1950 sur la côte de la mer Noire grâce à la possession de la plupart des établissements de cure et la 
politique de création d'une des "bases nationales de promotion de la santé populaire " (Nabieva et alii, 1997, p. 455). En 1961 le gouvernement crée le Grand Sotchi (l'ensemble des stations d'Adler à Touapsé) qui couvre $3500 \mathrm{~km}^{2}$ divisés en quatre districts plus ou moins urbanisés avec l'objectif de coordonner l'organisation de cette vaste zone de loisirs et de cure et d'utiliser au mieux les ressources naturelles et curatives du littoral de la mer Noire. Plus tard, les Soviétiques obtiennent le droit d'acheter leurs séjours en sanatorium ${ }^{6}$ ( "bon de séjour» ») ou les voyages en groupe.

Le pouvoir soviétique avait mis en place un système très strict pour les congés des citoyens : une fois par an, les administrations délivraient à chaque employé un «bon de séjour» lui permettant de partir pendant plusieurs semaines se reposer dans un des centres dépendant de l'entreprise pour laquelle il travaillait, sur le Baïkal, la Baltique ou encore au bord de la mer Noire, dans la région du Caucase ou en Crimée. La particularité du tourisme intérieur des années 1960 est l'orientation des séjours dans les stations balnéaires réservées autrefois aux séjours médicalisés vers des vacances qui comprennent du repos, du divertissement, du jeu. La progression du niveau de vie va contribuer à l'élaboration de la politique nationale de mobilité vacancière. L'État s'efforce de construire, aux endroits les plus réputés, mais également dans des paysages nouvellement valorisables, des équipements d'accueil pour les salariés bénéficiant des congés pays. Ainsi, à partir de 1967 le Grand Sotchi ${ }^{8}$ sera doté d'un plan directeur pour planifier l'implantation des établissements touristiques, organiser une administration unique des transports locaux et des excursions et développer un centre de recherches médicales sur les cures et la balnéothérapie (Cabanne, 2002, p. 237).

Pendant la période soviétique, nombreux étaient également les touristes qui se rendaient sur le littoral de la mer Noire par leurs propres moyens en utilisant les transports réguliers et se logeaient chez l'habitant en contactant directement des agents du "secteur privé» (sous-location d'appartements, camping, etc.) et en essayant individuellement de parvenir à un accord avec eux. En 1989, $62 \%$ des dépenses des touristes à Sotchi venaient de ces touristes individuels. Ce type de tourisme dikii ou « sauvage » est devenu majoritaire dans les années 1980 en Crimée et dans le Caucase, qui deviennent pendant cette période des endroits de repos pour les masses. Ainsi en 1988, la fréquentation touristique de Sotchi s'élève à 2,4 millions de touristes (Comité du tourisme de Sotchi).

\section{Tourisme et transition}

La chute de l'Union soviétique en 1991 a provoqué une crise politique, économique et sociale de la Russie. Cette dernière a réduit considérablement la pression touristique mais il s'agit d'une situation transitoire.

Sotchi, à la différence de Yalta en Crimée, n'a pas perdu sa notoriété après la chute de l'Union soviétique et la transition postsoviétique (Kanevsky, 2003) même si cette station balnéaire a subi des conséquences de la difficulté du tourisme à entrer dans l'économie du marché avec la baisse de sa fréquentation touristique entre 1991 (un peu plus de 2 millions de touristes) et 1998 (569 358) (Comité du tourisme de Sotchi). Suite au démantèlement de l'Union soviétique, la ville de Sotchi a subi un relâchement, la station semblait être abandonnée, l'infrastructure mal entretenue. Dès l'année 1992, la privatisation de certains hôtels et sanatoriums commence. Malgré la privatisation, le rôle des grandes administrations dans la région reste important. C'est le cas de la municipalité 
de Sotchi pour l'hôtel Radisson ou du Comité des biens de l'État dans le complexe de Dagomys et l'hôtel La Perla. Les autorités fédérales ou municipales ont gardé certaines participations dans les entreprises privées, la pratique devenue légale avec la mise en place de l'arrêté N813 du 25 avril 1994 intitulé «La mise en place des mesures complémentaires pour le développement du tourisme en Fédération de Russie et la règlementation de l'utilisation de la propriété de l'État dans le domaine du tourisme ", signé par le président Boris Eltsine. En 2000, dans la ville de Sotchi, $85 \%$ des entreprises sont privées mais l'État garde le contrôle de $2,4 \%$ et la municipalité de $3 \%$ d'entre elles (Cabanne, 2002). Pour faciliter le passage des entreprises touristiques d'un système économique à l'autre, un Comité du tourisme a été créé à la mairie de Sotchi le $1^{\text {er }}$ mars 1994 (Cabanne, 1997, p. 166).

Pendant la période de 1992 à 1998 les touristes continuaient à venir à Sotchi essentiellement à l'aide de vouchers délivrés par leurs entreprises, ce qui rendait leurs séjours totalement ou partiellement gratuits. Nous sommes donc encore dans cette logique du tourisme social héritée de l'époque soviétique. Très peu dépensiers et peu exigeants sur la qualité des prestations proposées sur place, ils freinent le développement économique du tourisme dans la région.

51 Lorsque la crise financière éclate en Russie en août $1998^{\circ}$, l'État se retrouve dans une situation plus que critique puisque son budget se trouve être déficitaire et il est donc incapable d'assainir les dépenses publiques. Néanmoins, cette crise sera salutaire pour le développement du tourisme intérieur grâce à l'adaptation des produits touristiques au marché et au contexte économique. Dès 1999 la reprise est forte pour la ville de Sotchi avec l'arrivée de 1,5 million de touristes dont 95,5\% de Russes (Comité du tourisme de Sotchi).

Quant à l'élite politique russe, c'est après la chute de l'URSS en 1991 que les dirigeants ont quitté la Crimée rattachée à l'Ukraine et se sont installés sur les rives du Caucase russe, à Sotchi. Construite en 1934, la seule datcha officielle russe sur le littoral de la mer Noire, nommée « Bocharov Ruchey », obtient le statut de la résidence présidentielle après 1991.

\section{Diversification, modernisation et adaptation de la station}

53 À partir des années 2000, les autorités politiques commencent à comprendre que si la Russie veut s'ouvrir à l'international, elle doit être formée au tourisme. Il n'est pas question de création mais de conversion. Il faut repenser le modèle de la période soviétique en tenant compte de l'ouverture à l'international.

\section{La reprise en main du tourisme par la politique et la transformation d'un lieu anciennement destiné au tourisme intérieur}

La région de Sotchi est en train de devenir la "Côte d'Azur russe », par la volonté du Kremlin. Au début des années 2000, avec l'arrivée au pouvoir de Vladimir Poutine, l'État russe commence vraiment à s'intéresser à la région de Krasnodar et plus particulièrement à la ville de Sotchi. C'est important de souligner dans cette analyse que le président russe Vladimir Poutine a un vrai faible pour cette ville. Il y passe du temps dans sa résidence présidentielle d'été. La ville de Sotchi devient la «capitale d'été » russe, de nombreuses 
rencontres officielles s'y déroulent. L'élite politique et d'affaires russe suivent le mouvement en organisant à Sotchi un grand nombre de congrès, séminaires, conférences. C'est également à Sotchi que se déroule tous les ans depuis 1991 le festival de cinéma Kinotavr (connu comme Sotchi Open Russian Film Festival), le plus grand festival de cinéma de Russie, considéré comme le festival national de Russie.

Sotchi bénéficie d'une approche spécifique dans la politique étatique du développement du tourisme intérieur en Russie, avec le programme fédéral couvrant la période 2006-2014 nommé « Développement de la ville de Sotchi en tant que station climatique ", qui vient en complément des investissements pour la préparation des échéances de Sotchi en 2014. Les 30 milliards de dollars dépensés pour les Jeux olympiques serviront selon Vladimir Poutine «à ce que des hommes et des femmes du monde entier visitent la Russie». Il existe un souhait au plus haut niveau de l'État d'ouvrir le tourisme à l'international. Par contre, nous émettons ici l'hypothèse que les travaux de construction d'infrastructures olympiques expriment principalement la volonté du gouvernement d'améliorer la cote de popularité du pays, et de faire oublier l'image négative de la politique russe à l'égard du reste du monde. Certes, les Jeux olympiques d'hiver de 2014 vont attirer un grand nombre de personnes vers la ville de Sotchi, mais nous pensons que juste après 2014 l'intérêt envers cette station balnéaire russe risque de baisser.

\section{Pour le développement et la diversification du tourisme à Sotchi : connivence des initiatives publiques et privées}

Les stations comme celle de Sotchi sont dépendantes des fluctuations météorologiques, elles souffrent de leur saisonnalité. La station de Sotchi se transforme progressivement depuis 10 ans en diversifiant son offre, en proposant diverses activités selon les saisons et en attirant des clientèles différentes. Comme le slogan de Sotchi l'indique, «chez nous, l'accueil est chaleureux toute l'année » : l'été, les touristes viennent se reposer, profiter de la mer, du climat agréable et des sanatoriums, alors que l'hiver fait place à une clientèle qui pratique les sports d'hiver et qui recherche le calme de la montagne.

De plus, conscient de cette saisonnalité, le gouvernement russe soutient un certain nombre de projets visant à la réduction de ses effets en se fondant sur les nouvelles formes du tourisme. La problématique de la gestion de saisonnalité concerne l'ensemble des acteurs du développement du territoire et nécessite une coopération entre les autorités et les professionnels du tourisme, dans les domaines de l'aménagement, du logement, de la restauration et de l'offre de loisir. Dans le cas russe, on peut souvent parler de la présence d'une "frontière invisible » entre le secteur public et privé en prenant en compte le lien que maintiennent les entreprises privées comme Gazprom ${ }^{10}$ avec le pouvoir politique russe, l'État étant actionnaire majoritaire du groupe.

En 2001, le gouvernement russe soutient l'initiative de comité olympique russe et l'administration de la région de Krasnodar qui vise la création du complexe touristique sportif Krasnaia Poliana («La clairière rouge »). Dès l'année 2002, Gazprom, qui possédait déjà l'hôtel Radisson SAS Lazurnaya à Sotchi, s'était intéressé au projet de création des pistes de ski alpin et du village à Krasnaia Poliana. Le complexe Le Grand Hôtel Polyana (5 étoiles) construit par Gazprom a ouvert ses portes en février 2008 au pied du téléphérique. 

(comme Interros ${ }^{11}$ ou Umaco ${ }^{12}$ ) consistent en création d'hôtels de classe « luxe », c'est-àdire de plus de 5 étoiles de type luxury (boutique et hôtels), dont le premier est le Grand Hotel Spa Rodina ( «a Patrie ») ouvert à Sotchi en 2006 par l'oligarque Oleg Deripaski. Cet hôtel comprend 40 chambres, 2 restaurants, une bibliothèque, une salle de cinéma, une piscine, un court de tennis, un court de basket-ball, une plage privée. Les prestations de Grand Hotel Spa Rodina répondent aux attentes de la clientèle aisée mais ne pourront pas attirer les touristes de la classe moyenne.

Un autre projet devrait voir le jour: un port de plaisance avec un club de yacht de 300 places dans la ville de Dagomys, située à $13 \mathrm{~km}$ de Sotchi. Ce projet sera réalisé en quatres étapes qui s'étaleront sur 10 ans. L'infrastructure comprendra la location d'équipements, voile, plongée, canotage, ski nautique, aquarium, parc aquatique, un réseau d'hôtels 4 et 5 étoiles et un centre de loisirs pour un montant total de 703,5 millions de dollars (518 millions d'euros).

61 Un troisième projet concerne la création de 18 terrains de golf pour le Sotchi Golf Club dans la région d'Adler. Ce dernier comprend la construction d'un spa, d'un centre de sport, des piscines, d'un hôtel 4 étoiles et des centres commerciaux pour un montant total de 250 millions d'euros.

62 Enfin, le 22 juillet 2014, le président russe Vladimir Poutine a signé une loi portant sur la création de deux nouvelles zones de jeux d'argent à Sotchi et en Crimée, avec l'objectif d'élargir la gamme de services proposés aux touristes. L'emplacement de la zone de jeux sera choisi par le gouvernement. L'utilisation de sites olympiques financés par les investisseurs privés est autorisée par la loi russe (Lossan, 2014).

63 Grace à ces quelques exemples on voit comment la ville de Sotchi, se trouvant à proximité géographique de Moscou ( 2 heures d'avion seulement) devient de plus en plus la banlieue chic de Moscou. Les oligarques russes, les artistes, les sportifs y vont pour la mer et la montagne. Le problème est que tous ces projets sont pensés pour un seul type de clientèle. Le gouvernement russe vise peu la clientèle de la classe moyenne, malgré le fait qu'un développement du tourisme en profondeur semble devenir indispensable à la société russe. Il y a, par exemple, un manque réel d'hôtels de catégorie intermédiaire dans toutes les villes russes. Sotchi ne fait pas exception. La page dédiée au tourisme sur le site de la municipalité de Sotchi abrite le slogan suivant «Le repos à Sotchi est meilleur en Russie ». C'est un argument important pour attirer les touristes russes qui ne veulent pas partir à l'étranger. Néanmoins, ce « meilleur repos » semble ne pas être accessible à tous.

Les Russes aisés vont pouvoir continuer à profiter du dispositif des ZES mis en place par le pouvoir central. Par le biais de ce projet, l'État souhaite encourager les investisseurs qui voudraient développer le secteur privé sur place en leur offrant des avantages fiscaux et un environnement économique favorable. Ce favoritisme va continuer à provoquer une spéculation des terres non agricoles sur les côtes de la mer Noire comme ce fut le cas dans d'autres littoraux sur la Côte d'Azur ou en Espagne (la Costa Brava ou la Costa del Sol). Tout cela va entraîner la pérennisation de cette distinction sociale qui existe dans la société russe en ce qui concerne l'accès aux différentes pratiques touristiques au sein du pays. 


\section{Conclusion}

65

d'aménagement touristique et de gestion de saisonnalité, représente une sorte de miroir de la société russe actuelle et démontre l'existence d'un lien fort qui existe entre les recompositions territoriales et politiques dans le pays. D'un coté, ce lieu touristique de bord de mer Noire est d'une forte pérennité grâce à une certaine continuité des pratiques (soin, repos, découverte, jeux) qui le caractérise depuis plus de deux siècles. D'un autre coté, il ne faut pas nier le rôle de l'État dans le développement de ce territoire, car le littoral de la mer Noire, en tant que plus vaste domaine du tourisme côtier russe, est un lieu stratégique pour le pouvoir en place. L'organisation des Jeux olympiques d'hiver en 2014 et les nouveaux aménagements d'espaces en sont de bons exemples.

67 La thématique abordée dans cet article pourra être analysée à l'avenir sous l'angle de l'étude du tourisme intérieur en tant qu'élément contribuant à l'affirmation de l'identité nationale russe. Cette étude devrait être effectuée sous le prisme de la politique, élément indispensable pour la bonne compréhension des systèmes touristiques du pays.

\section{BIBLIOGRAPHIE}

\section{Archives et documents officiels}

Agence fédérale du tourisme de la Fédération de Russie, 2012.

Arrêté N 813 du 25 avril 1994, « La mise en place des mesures complémentaires pour le développement du tourisme en Fédération de Russie et la réglementation de l'utilisation de la propriété de l'État dans le domaine du tourisme ».

Arrêté N 833 du gouvernement de la Fédération de Russie du 14 octobre 2010, «Création sur le territoire de la région de Stavropol d'une zone économique spéciale ».

Comité du tourisme de Sotchi, rapports annuels (en russe).

Loi fédérale du 22 juillet 2005, N116-F3, « Les zones économiques spéciales en Fédération de Russie ».

Rosstat, Service fédéral des statistiques, 2011.

\section{Ouvrages}

Mikhail Bokov, Nadejda Demertchan, Svetlana Nefedkina et Eugene Ougroumov, Les Particularités du développement du tourisme régional en Russie, Éditions de l'université de Sotchi, 2012 (en russe). 
Claude Cabanne et Elena Tchistiakova, La Russie : perspectives économiques et sociales, Armand Colin, 2002.

Équipe Mit, Tourismes. 1, Lieux communs, « Mappemonde », Belin, 2002.

Équipe Mit, Tourismes. 3, La révolution durable, « Mappemonde », Belin, 2011.

\section{Articles de revue}

Claude Cabanne, « Les évolutions récentes du tourisme sur le littoral russe de la mer Noire », Cahiers nantais, $\mathrm{n}^{\text {os }} 47-48,1997$, p. 163-166.

Claude Cabanne, «Sotchi, capitale balnéaire de la Russie », Cahiers nantais, n 58, 2002, p. 235-241.

Ouma Nabieva, Elena Tchistiakova, Claude Cabanne et Alain Miossec, « Mutations touristiques et tourisme littoral en Russie », Norois, vol. 44, nº 175, 1997, p. 445-462 [http://www.persee.fr/doc/ noroi_0029-182x_1997_num_175_1_6807].

\section{Documents internet}

Natalia Kanevsky, « Yalta et Sotchi, deux visages d'une côte mythique », Regard sur l'Est, avril 2003 [http://www.regard-est.com/home/breve_contenu.php?id=368].

Alexeï Lossan, « Sotchi va construire des casinos pour concurrencer le tourisme en Crimée », Russia Beyond the Headlines, 26 juillet 2014 [http://fr.rbth.com/economie/2014/07/26/ sotchi_va_construire_des_casinos_pour_concurrencer_le_tourisme_en_cr_30157.html]. Irina Zoubareva, «Les zones qui attirent les résidents », Journal de Russie, 22 avril 2014 [http:// www.rg.ru/2014/04/22/startap.html].

Vadim Tretiakov, «Interview du P-DG de la société de gestion des ZES Vadim Tretiakov », RBK Daily, 23 janvier 2014 [http://rbcdaily.ru/economy/562949990326883].

\section{Thèse}

Ekaterina Andreeva, Les Pratiques touristiques des Russes en France. Analyse comparée du XIX siècle à nos jours, thèse de doctorat, université de Saratov et université d'Angers, 2006.

\section{NOTES}

1. Nous ne souhaitons pas porter une attention particulière aux Jeux olympiques dans cet article. Nous nous limiterons dans ce domaine, à la fin de cette analyse, à l'émission d'une hypothèse concernant l'impact de cet événement sportif au développement du tourisme intérieur et international dans la région de Sotchi.

2. L'estimation de la classe moyenne en Russie varie énormément en fonction des études et des moyens de comparaison choisis. Selon le ministère russe du Développement économique et du Commerce, la classe moyenne russe s'élèverait en 2011 à environ $22 \%$ de la population.

3. L'Anneau d'or est un espace situé au Nord-Est de Moscou entre la Volga et la Kliazma et délimité par d'anciennes cités princières : Vladimir, Souzdal, Iaroslavl, etc.

4. Grande ferme d'État en URSS.

5. Exploitation agricole collective en URSS.

6. Il s'agit d'une maison de cure ou de repos.

7. Poutiovka en russe.

8. En 1967, le Grand Sotchi comprend huit stations de cure (Magri, Ache, Lazarevskoye, Golovinka, Dagomys, Sotchi-centre et Adler). On y rajoutera Krasnaia Poliana destinée à devenir une importante base de tourisme et de sports d'hiver. 
9. Dévolution du rouble, l'inflation en Russie est de $84 \%$.

10. Le premier producteur et exportateur de gaz dans le monde

11. Grand groupe industriel russe

12. Compagnie de gestion d'hôtels

\section{RÉSUMÉS}

En Russie postsoviétique, les stations balnéaires du littoral de la mer Noire constituent un laboratoire d'observation de tendances plus générales permettant de comprendre les mutations au sein du pays. Dans cet article nous proposons d'analyser le processus de la construction de la pérennité d'un lieu touristique comme Sotchi, et d'étudier les transformations de la station sous le prisme des nouvelles formes de tourisme et des investissements des acteurs publics et privés. L'examen de ce cas particulier nous permettra de tirer des conclusions plus générales sur le positionnement des lieux dans le champ du tourisme, tout en prenant en compte les spécificités d'accès au tourisme d'un pays dit « émergent » comme la Russie.

Black Sea coasts in post-Soviet Russia reflect more general trends within the country. They help to understand the changes all over the country. We propose to analyze in this article the process of building of the sustainability of a tourist place like Sochi and to explore the transformations of the station through the survey of some new forms of tourism and investment of public and private actors. The examination of this case will allow us to draw more general conclusions about the positioning of the places in the tourism field considering the specificities of access to tourism of an "emerging" country like Russia.

\section{INDEX}

Mots-clés : Russie, Sotchi, durabilité, tourisme domestique, État

Keywords : Russia, Sochi, sustainability, domestic tourism, state

\section{AUTEUR}

\section{EKATERINA ANDREEVA-JOURDAIN}

Chercheuse associée à l'UMR 6590, ESO Angers - MCF à l'UFR ITBS/Imis-Esthua, université

d'Angers, 7, allée François Mitterrand - BP 40455 - 49004 Angers cedex 01 -

ekaterina.jourdain@univ-angers.fr 